November 2, 2018

\title{
A Lattice-Boltzmann method for the simulation of transport phenomena in charged colloids
}

\author{
Jürgen Horbach * and Daan Frenkel \\ Kruislaan 407, 1098 SJ Amsterdam, Netherlands
}

\begin{abstract}
We present a new simulation scheme based on the Lattice-Boltzmann method to simulate the dynamics of charged colloids in an electrolyte. In our model we describe the electrostatics on the level of a Poisson-Boltzmann equation and the hydrodynamics of the fluid by the linearized Navier-Stokes equations. We verify our simulation scheme by means of a Chapman-Enskog expansion. Our method is applied to the calculation of the reduced sedimentation velocity $U / U_{0}$ for a cubic array of charged spheres in an electrolyte. We show that we recover the analytical solution first derived by Booth (F. Booth, J. Chem. Phys. 22, 1956 (1954)) for a weakly charged, isolated sphere in an unbounded electrolyte. The present method makes it possible to go beyond the Booth theory, and we discuss the dependence of the sedimentation velocity on the charge of the spheres. Finally we compare our results to experimental data.

PACS numbers: 47.65.+a, 82.70.Dd, 47.11.+j, 83.85.Pt, 05.20.Dd, 07.05.Tp, $66.20 .+\mathrm{d}$
\end{abstract}

*Author to whom correspondence should be addressed. e-mail: horbach@amolf.nl 


\section{INTRODUCTION}

The simulation of the dynamics of colloidal suspensions is a challenging task. The reason is that the movement of the colloidal particles can be on a time scale which is orders of magnitudes slower than that of the solvent particles (e.g. seconds versus picoseconds). Therefore, simulation methods such as Molecular Dynamics, that account for the fluid particles explicitly, are not well suited to study the dynamics of colloidal suspensions because one would spend most of the simulation time solving the equations of motion of the fluid particles. The situation for charged colloids is even worse because one has to take into account the long-ranged Coulomb interactions which are already very time consuming in simple ionic liquids.

One possibility to circumvent these problems is to avoid explicit simulation of the solvent particles and describing the interaction between the colloidal particles by means of an effective potential. In the case of charged colloidal systems this is usually a Yukawa-like potential which gives in many cases quite an accurate description of interaction-dependent properties [1,2]. But the approach with effective interactions neglects completely the hydrodynamic interactions between the colloidal particles which stem from the momentum transport through the solvent. In order to take this into account one has to treat the hydrodynamics of the solvent at least on a coarse-grained level. An effective scheme, that was developed to solve efficiently the Navier-Stokes equations, is the so-called lattice Boltzmann method (LBM). The LBM is a pre-averaged version of a lattice gas, i.e., a Boltzmann equation is solved on a lattice such that the Navier-Stokes equations are recovered (reviews of the method are the Refs. [3 5]). Recently, the LBM was applied to simulate the dynamics of colloidal systems, such as the rotational and translational short-time dynamics of colloids [6 10, the diffusion of colloidal particles in confined geometry [11 [13], and the dynamics in porous media [14 [17]. Also other complex systems like polymer solutions [18] have been investigated by LBM.

Several attempts to apply the LBM to charged systems have been reported in the literature. He and Li [19] proposed a scheme which is appropriate to study electrochemical processes in an electrolyte. However, in this method it is assumed that the fluid is locally electrically neutral which cannot be true for the part of an electrolyte forming the electrical double layer around a macroion. Thus, the LBM of He and Li cannot be used to describe the dynamics of suspensions of charged colloids. A different LBM for charged systems was suggested by Warren [20]. The central idea of this method is the introduction of external charge densities $\rho_{s}$ for the ionic species of type $s$ which are propagated with the one particle distribution function of the LB equation by means of the so-called moment propagation method [21]. These ionic densities are coupled back to the mass current of the LB equation via a chemical potential which consists of a term $\propto \ln \rho_{s}$ and a term proportional to the electrostatic potential such that it only gives a contribution if the ion densities are not distributed as expected in equilibrium by a Boltzmann distribution (e.g. because of an external electrical field). Finally, the electrostatic potential is determined from the charge densities

by means of a Poisson equation solver. The main drawback of Warren's method is that the charge densities are introduced as additional physical quantities which are independent from the mass density in the LB equation, and thus, this method is not self-consistent and the correctness of coupling of the charge density to the mass current is not obvious. 
Our LBM for charged colloidal suspensions is inspired by Warren's approach, but it does not introduce ionic species as additional quantities. The details of our method can be found in the following sections. We apply it to the determination of the sedimentation velocity of an array of charged spheres in an electrolyte.

The article is organized as follows: In Sec. II we give a brief introduction into the electrokinetic equations of motion with which we model the dynamics of the fluid. In Sec. III the LBM is presented which solves the latter equations of motion on a lattice. The LBM is verified in Sec. IV by means of a Chapman-Enskog expansion. And in Sec. V we show our results for the sedimentation phenomena. We conclude with a discussion of the method and the results.

\section{THE ELECTROKINETIC EQUATIONS}

In this section we introduce the equations of motion for a hydrodynamic description of charged colloidal suspensions. These equations can be found in standard textbooks (see e.g. Ref. [22]).

Consider a system of macroions with radius $a$ in an electrolyte consisting of two ionic species which have charges $+z_{1} e$ and $-z_{2} e$, respectively (where $z_{1}$ and $z_{2}$ are the valencies of the ions and $e$ is the charge of a proton). The densities of the ions, $\rho_{1}(\vec{r}, t)$ and $\rho_{2}(\vec{r}, t)$, are conserved quantities and therefore each of them follows a continuity equation:

$$
\frac{\partial \rho_{s}}{\partial t}=-\nabla \cdot \vec{J}_{s} \quad s=1,2
$$

The current $\vec{J}_{s}$ is given by

$$
\overrightarrow{J_{s}}=\rho_{s} \vec{u}-D_{s} \nabla \rho_{s}-z_{s} D_{s} \rho_{s} \nabla \hat{\Phi} .
$$

The first term in (2) in which $\vec{u}$ denotes the flow velocity is the convection current whereas the two other terms describe the diffusive current and the current due to the electrostatic potential $\Phi . D_{s}$ denotes the diffusivity of ions of type $s$ and $\hat{\Phi}$ is the electrostatic potential in dimensionless form, $\hat{\Phi}=\frac{e}{k_{B} T} \Phi\left(k_{B}\right.$ : Boltzmann constant, $T$ : temperature).

$\hat{\Phi}$ is determined by the Poisson equation,

$$
\nabla^{2} \hat{\Phi}=-4 \pi l_{B}\left(\sum_{s=1}^{2} z_{s} \rho_{s}+\sigma\right)
$$

where the Bjerrum length $l_{B}$ is defined by

$$
l_{B}=\frac{e^{2}}{4 \pi \epsilon k_{B} T}
$$

$\epsilon$ is the dielectric constant. At a distance $l_{B}$ the Coulomb energy of one ion due to another ion is equal to $k_{B} T$. $\sigma$ denotes the charge density of the macroion. We assume that the charge $Z$ of each macroion sits on its surface in the form of uniformly distributed point charges. 
If we set $\vec{u}=0$ in Eqs. (1/1) and (2) we yield the equilibrium solution for the ion densities as

$$
\rho_{s}(\vec{r})=\bar{\rho}_{s} \exp \left(-z_{s} \hat{\Phi}(\vec{r})\right) .
$$

By putting the Boltzmann distribution (5) into the Poisson equation (3) this leads to the so-called Poisson-Boltzmann equation in which correlations between the ions are neglected. Moreover, if one linearizes the exponential function in Eq. (5) (Debye-Hückel theory) it is possible to solve Eq. (3) analytically and the result is a Yukawa potential,

$$
\hat{\Phi}(\vec{r})=K \frac{\exp \left(-|\vec{r}| / \lambda_{D}\right)}{|\vec{r}|} .
$$

The so-called Debye length is defined by

$$
\lambda_{D} \equiv \kappa^{-1}=\frac{1}{\sqrt{4 \pi l_{B} \sum_{s} z_{s}^{2} \bar{\rho}_{s}}} .
$$

The potential (6) is even a good approximation in the non-linear case but the prefactor $K$ is then different from the one of the linear theory.

The equation of motion which has to be specified finally is that for the total mass current of the fluid, $\rho \vec{u} \equiv\left(\sum_{s=1}^{2} \rho_{s}+\rho_{n}\right) \vec{u}$, where $\rho_{n}$ is the density of the neutral part of the fluid. We assume that our fluid can be described by the linearized Navier-Stokes equations for low Reynolds number flow. Hence, the equation for $\rho \vec{u}$ with a body force due to the electrostatic potential is

$$
\frac{\partial(\rho \vec{u})}{\partial t}=\nu \nabla^{2} \rho \vec{u}-\nabla p-k_{B} T \sum_{s} z_{s} \rho_{s} \nabla \hat{\Phi}
$$

where $p$ is the pressure and $\nu$ is the kinematic viscosity. If the equation for the pressure is the one of an ideal gas, $p=k_{B} T \rho, p$ can be decomposed into an electrostatic and a neutral part, $p_{e}=k_{B} T \sum_{s=1}^{2} \rho_{s}$ and $p_{n}=k_{B} T \rho_{n}$, respectively. The sum of $-\nabla p_{e}$ and the electrostatic body force, i.e. the last term in Eq. (8), is zero, if the ion densities have relaxed to their equilibrium distribution, Eq. (5).

\section{THE LATTICE-BOLTZMANN METHOD FOR CHARGED COLLOIDS}

We have developed a simulation method to solve the non-linear coupled Eqs. (11), (3), and (8) numerically. For this we use concepts which are well-known from the LBM.

In this method the discretized version of a Boltzmann equation is solved numerically on a lattice on which every lattice point represents a cell of particles. The central quantity is the one-particle distribution function $n_{i}(\vec{r}, t)$ which describes the number of particles on a lattice node $\vec{r}$ at time $t$ with a discrete velocity $\vec{c}_{i}$. The discrete space of velocities $\left\{\vec{c}_{i}\right\}$ is chosen such that no artificial anisotropic terms appear in the corresponding equations in the continuous limit. In our case the velocity space consists of 18 vectors of which from a given lattice node 6 point to the nearest and 12 to next-nearest neighbors on a simple cubic lattice. This velocity space can be constructed by projecting the unit vectors of a 
four dimensional FCHC lattice onto three dimensions. It is one possible choice of a velocity space which exhibits the required isotropy.

The equation of motion for $n_{i}(\vec{r}, t)$ consists of two steps, a collision and a propagation step. In the collision step the interaction between the particles is taken into account which results in the post-collision function $n_{i}^{\star}\left(\vec{r}, t^{\star}\right)$ at the collision time $t^{\star}$. In the propagation step $n_{i}(\vec{r}, t)$ is updated by

$$
n_{i}\left(\vec{r}+\vec{c}_{i}, t+1\right)=n_{i}^{\star}\left(\vec{r}, t^{\star}\right) .
$$

In this equation the lattice constant, the time step, and the mass of a particle is set to unity. The density $\rho(\vec{r}, t)$ and the mass current $\vec{j} \equiv \rho \vec{u}$ are given by the zeroth and first moment of $n_{i}$, respectively, $\rho=\sum_{i} n_{i}(\vec{r}, t), \vec{j}=\sum_{i} n_{i}(\vec{r}, t) \vec{c}_{i}$.

In the case of the charged system a one-particle distribution function for each ion species and a neutral part is required. The purpose of the neutral part is to keep the viscosity essentially constant through the fluid. Thus, it is chosen such that its value at a given lattice point is much higher than that of the ionic densities. We make the following Ansatz for the post-collision function $n_{i}^{s \star}$ for the counter- and coions, $s=+,-$, respectively, and the neutral part, $s=n$ (we also take into account rest particles by the index $i=0$ ):

$$
\begin{aligned}
& n_{i}^{s \star}\left(\vec{r}, t^{\star}\right)=\frac{\mathrm{w}_{i} \gamma_{s}}{24} \rho_{s}(\vec{r}, t)\left(1+\frac{1}{c_{\mathrm{sv}}^{2} \rho^{\prime}(\vec{r}, t)} \vec{j}(\vec{r}, t) \cdot \vec{c}_{i}\right), \\
& n_{0}^{s \star}\left(\vec{r}, t^{\star}\right)=\left(1-\gamma_{s}\right) \rho_{s}(\vec{r}, t) .
\end{aligned}
$$

The factor $\mathrm{w}_{i}$ is a weighting factor which is equal to 2 for the $\vec{c}_{i}$ in the direction of nearest neighbors and equal to 1 for the remaining $\vec{c}_{i}$. So it satisfies the normalization constraint $\sum_{i=1}^{18} \frac{\mathrm{w}_{i}}{24}=1$. For the following we define also $\mathrm{w}_{0}:=0$. With the parameter $0<\gamma_{s} \leq 1$ the diffusivity $D_{s}$ of the particles of type $s$ can be varied. The latter quantity is given by

$$
D_{s}=\frac{c_{\mathrm{sv}}^{2}}{2} \gamma_{s}
$$

which is shown in the next section. $c_{\mathrm{Sv}}$ is the sound velocity which is $1 / \sqrt{2}$ for our model [4]. The density $\rho^{\prime}$ is defined by $\rho^{\prime}=\sum_{s} \gamma_{s} \rho_{s}$.

The propagation step for our charged system is

$$
\begin{aligned}
n_{i}^{s}\left(\vec{r}+\vec{c}_{i}, t+1\right) & =n_{i}^{s \star}\left(\vec{r}, t^{\star}\right), \\
n_{0}^{s}(\vec{r}, t+1) & =n_{0}^{s \star}\left(\vec{r}, t^{\star}\right) .
\end{aligned}
$$

Different propagation rules have to be established at the surface of the macroions and at walls. Here we use the bounce back rules suggested by Ladd [4] which lead to no-slip boundary conditions. In this scheme one puts a sphere which represents a macroion onto the lattice whereby its surface cuts links between lattice nodes. The boundary nodes are defined halfway along these links and the population functions $n_{i}^{s}$ which point to the direction of the boundary nodes are reflected back during the propagation step. In the case of moving boundaries there is a momentum transfer between the boundary nodes and the fluid. In this case the self consistent scheme derived by Lowe et al. [9, [10] can be used.

The aforementioned way of mapping a sphere onto the lattice introduces fluid inside and outside the sphere. In our scheme we assign the charge of the macroions in that the inner 
fluid is an electrolyte with net charge $Z$. Charge neutrality requires that the total charge of the outer fluid equals the one of the inner fluid of the macroions. Of course, it is not allowed in our scheme that outer fluid leaks through the surface of the sphere. Therefore, only small movements of a macroion are possible such that the center of mass of the sphere can be fixed, and only a momentum transfer with the fluid takes place.

The densities $\rho_{s}$ and the total mass current $\vec{j}$ cannot be inferred simply from the zeroth and first moments of the $n_{i}^{s}$ 's because we have to take into account their coupling to the gradient of the electrostatic potential. If $\rho_{s}$ and $\vec{j}$ are calculated as follows,

$$
\rho_{s}(\vec{r}, t+1)=\sum_{i=0}^{18}\left(n_{i}^{s}(\vec{r}, t+1)-\frac{z_{s}}{2} \frac{\mathrm{w}_{i} \gamma_{s}}{24} \rho_{s}\left(\vec{r}-\vec{c}_{i}, t\right) \nabla \hat{\Phi}\left(\vec{r}-\vec{c}_{i}, t\right) \cdot \vec{c}_{i}\right)
$$

and

$$
\vec{j}(\vec{r}, t+1)=\sum_{s=1}^{3}\left(\sum_{i=1}^{18} n_{i}^{s}(\vec{r}, t+1) \vec{c}_{i}-c_{\mathrm{sv}}^{2} z_{s} \gamma_{s} \rho_{s}(\vec{r}, t+1) \nabla \hat{\Phi}(\vec{r}, t+1)\right) .
$$

we are consistent with Eqs. (11) and (8) in the continuous limit. $\nabla \hat{\Phi}$ does not couple to the neutral part of the fluid. This is guaranteed in Eqs. (15) and (16) by setting $z_{s}=0$ for $s=n$.

If one replaces $n_{i}^{s}(\vec{r}, t+1)$ by $n_{i}^{s}\left(\vec{r}-\vec{c}_{i}, t\right)$ in Eqs. (15) and (16) by using Eq. (13) it becomes clear that Eqs. (15) and (16) are the discrete versions of Eqs. (11) and (8), respectively. Thereby, the second terms in Eqs. (15) and (16) correspond respectively to the current due to the electrostatic potential in Eq. (11) and the electrostatic body force in Eq. (8). We show this explicitly in the next section by means of a Chapman-Enskog expansion. Of course, with the $\nabla \hat{\Phi}$ terms in Eqs. (15) and (16) the conservation of the ionic densities and the mass current is still fulfilled. This is guaranteed because $\hat{\Phi}$ is determined self-consistently from the ionic densities by means of the Poisson equation.

This means that we have to solve the Poisson equation at each time step in order to determine the electrostatic potential from the ionic densities $\rho_{+}$and $\rho_{-}$. For this purpose we use a successive over-relaxation (SOR) scheme in which one looks in principle for the stationary solution of a diffusion equation 23. But in contrast to a normal diffusion equation one introduces an acceleration parameter $1<\omega<2$ such that the potential is obtained as the iterative solution of the following equation:

$$
\hat{\Phi}_{h+1}(\vec{r}, t)=\omega\left[\sum_{i}\left(\frac{\mathrm{w}_{i}}{24} \hat{\Phi}_{h}\left(\vec{r}-\vec{c}_{i}, t\right)+4 \pi l_{B} \frac{c_{\mathrm{SV}}^{2}}{2} \sum_{s=+,-} z_{s} \rho_{s}(\vec{r}, t)\right)\right]+(1-\omega) \hat{\Phi}_{h}(\vec{r}, t) .
$$

In this equation we have denoted the iteration time by $h$ (the unit for an iteration step is again set to unity). For a sufficient number of iteration steps $\Phi_{h}$ converges to $\Phi$. We have found that a suitable choice for $\omega$ is 1.45 which guarantees stability and optimal acceleration. The gradient of $\hat{\Phi}$ which we need in Eqs. (15) and (16) is given by

$$
\nabla \hat{\Phi}(\vec{r})=-\sum_{i} \frac{\mathrm{w}_{i}}{24 c_{\mathrm{sv}}^{2}} \hat{\Phi}\left(\vec{r}-\vec{c}_{i}\right) \vec{c}_{i}
$$

Note that we use in Eqs. (17) and (18) the same discrete space as the velocity space in the Lattice-Boltzmann equations. This means that the truncation error which is caused due 
to the discrete representation of the derivatives is of fourth order in contrast to a second order truncation error on a simple cubic lattice with 6 vectors $\left\{\vec{c}_{i}\right\}$ pointing to the nearest neighbors from a given lattice node. The stability and efficiency of the SOR algorithm are further optimized by making use of a partially decoupled red-black Gauss-Seidel scheme [24.

\section{CHAPMAN-ENSKOG EXPANSION}

By means of a Chapman-Enskog expansion we show in this section that the set of discrete equations from the previous section indeed recover the Eqs. (1), (3), and (8) in the continuous limit.

As a first step we rewrite Eqs. (15) and (16) for the densities $\rho_{s}$ and the partial currents $\overrightarrow{j_{s}} \equiv \gamma_{s} \rho_{s} \vec{j} / \rho^{\prime}$ :

$$
\begin{aligned}
\rho_{s}(\vec{r}, t)= & \sum_{i} \frac{\mathrm{w}_{i} \gamma_{s}}{24}\left(\rho_{s}\left(\vec{r}-\vec{c}_{i}, t-1\right)+\vec{j}_{s}\left(\vec{r}-\vec{c}_{i}, t-1\right) \cdot \vec{c}_{i}\right. \\
& \left.+\frac{z_{s}}{2} \rho_{s}\left(\vec{r}-\vec{c}_{i}, t-1\right) \nabla \hat{\Phi}\left(\vec{r}-\vec{c}_{i}, t-1\right) \cdot \vec{c}_{i}\right), \\
\vec{j}_{s}(\vec{r}, t)= & \sum_{i} \frac{\mathrm{w}_{i} \gamma_{s}}{24}\left(\rho_{s}\left(\vec{r}-\vec{c}_{i}, t-1\right) \vec{c}_{i}+\vec{j}_{s}\left(\vec{r}-\vec{c}_{i}, t-1\right) \cdot \vec{c}_{i} \vec{c}_{i}\right) \\
& -c_{\mathrm{sv}}^{2} \gamma_{s} z_{s} \rho_{s}(\vec{r}, t) \nabla \hat{\Phi}(\vec{r}, t) .
\end{aligned}
$$

If we now expand the functions of the form $f\left(\vec{r}-\vec{c}_{i}, t-1\right)\left(f=\rho_{s}, \vec{j}_{s}, \rho_{s} \nabla \hat{\Phi}\right)$ around position $\vec{r}$ and time $t$ up to second order,

$$
f\left(\vec{r}-\vec{c}_{i}, t-1\right)=f(\vec{r}, t)+\left(-\partial_{t}-c_{i \alpha} \nabla_{\alpha}+\frac{1}{2}\left(\partial_{t}+c_{i \alpha} \nabla_{\alpha}\right)^{2}\right) f(\vec{r}, t)
$$

we obtain the following equations for $\rho_{s}$ and $\vec{j}_{s}$,

$$
\begin{aligned}
& \partial_{t} \rho_{s}=\frac{1}{2} \partial_{t}^{2} \rho_{s}-\nabla \cdot \vec{j}_{s}+\partial_{t} \nabla \cdot \vec{j}_{s}+\frac{c_{\mathrm{sv}}^{2}}{2} \gamma_{s}\left(\nabla^{2} \rho_{s}+z_{s} \nabla \cdot \rho_{s} \nabla \hat{\Phi}+z_{s} \partial_{t} \nabla \cdot \rho_{s} \nabla \hat{\Phi}\right) \\
& \partial_{t} \vec{j}_{s}=-c_{\mathrm{sv}}^{2} \gamma_{s} \nabla \rho_{s}+c_{\mathrm{sv}}^{2} \gamma_{s} \partial_{t} \nabla \rho_{s}+\frac{1}{2} \partial_{t}^{2} \vec{j}_{s}+\frac{1}{6} \nabla^{2} \vec{j}_{s}-c_{\mathrm{sv}}^{2} \gamma_{s} z_{s} \rho_{s} \nabla \hat{\Phi} .
\end{aligned}
$$

To achieve Eqs. (22) and (23) we have used the lattice sums [4]

$$
\begin{aligned}
\sum_{i=1}^{18} c_{i \alpha} c_{i \beta} & =c_{\mathrm{sv}}^{2} \delta_{\alpha \beta} \\
\sum_{i=1}^{18} c_{i \alpha} c_{i \beta} c_{i \gamma} c_{i \delta} & =\frac{1}{3}\left(\delta_{\alpha \beta} \delta_{\gamma \delta}+\delta_{\alpha \gamma} \delta_{\beta \delta}+\delta_{\alpha \delta} \delta_{\beta \gamma}\right) .
\end{aligned}
$$

Eqs. (22) and (23) are the starting point for the Chapman-Enskog expansion which introduces a macroscopic space scale by $\vec{r}_{1}=\epsilon \vec{r}$ and two macroscopic time scales by $t_{1}=\epsilon t$ and $t_{2}=\epsilon^{2} t$. The $t_{1}$ scale describes fast convection processes whereas on the slower $t_{2}$ scale the diffusion of vorticity takes place. Thus, the Chapman-Enskog expansion enables us to 
consider the latter time scales separately. It was for the first time applied to lattice gases by Frisch et al. [25].

We now express the derivatives by means of $\vec{r}_{1}, t_{1}$, and $t_{2}$,

$$
\begin{aligned}
& \nabla=\epsilon \nabla_{1} \\
& \partial_{t}=\epsilon \partial_{t_{1}}+\epsilon^{2} \partial_{t_{2}},
\end{aligned}
$$

and put them into Eqs. (22) and (23). If we collect terms of the same order in $\epsilon$ we obtain on the $\epsilon^{1}$ scale

$$
\begin{aligned}
& \partial_{t_{1}} \rho_{s}=-\nabla_{1} \cdot \vec{j}_{s} \\
& \partial_{t_{1}} \vec{j}_{s}=-c_{\mathrm{sv}}^{2} \gamma_{s}\left(\nabla_{1} \rho_{s}+z_{s} \rho_{s} \nabla_{1} \hat{\Phi}\right) .
\end{aligned}
$$

Eq. (28) is the continuity equation for mass conservation. If one takes the sum over $s$ on both sides of Eq. (29) one obtains the "fast" part of the linearized Navier-Stokes equation for the total mass current $\vec{j}$ :

$$
\partial_{t_{1}} \vec{j}=-c_{\mathrm{sv}}^{2} \sum_{s}\left(\nabla \rho_{s}+z_{s} \rho_{s} \nabla_{1} \hat{\Phi}\right)
$$

The first term on the right-hand side of this equation is the negative gradient of the pressure and the second term the electrostatic body force.

On the $\epsilon^{2}$ scale we have

$$
\begin{aligned}
& \partial_{t_{2}} \rho_{s}=\frac{1}{2} \partial_{t_{1}}^{2} \rho_{s}+\partial_{t_{1}} \nabla_{1} \cdot \vec{j}_{s}+\frac{c_{\mathrm{sv}}^{2}}{2} \gamma_{s}\left(\nabla_{1}^{2} \rho_{s}+z_{s} \nabla_{1} \cdot \rho_{s} \nabla \hat{\Phi}\right), \\
& \partial_{t_{2}} \vec{j}_{s}=c_{\mathrm{sv}}^{2} \gamma_{s} \partial_{t_{1}} \nabla_{1} \rho_{s}+\frac{1}{2} \partial_{t_{1}}^{2} \vec{j}_{s}+\frac{1}{6} \nabla^{2} \vec{j}_{s} .
\end{aligned}
$$

From Eqs. (28)-(32) we see that the transport of the ion densities to equilibrium can be either achieved by convection or by diffusion. So, if $\partial_{t_{1}} \vec{j}_{s}$ vanishes Eq. (29) is solved by the Boltzmann distribution, Eq. (5). And by using Eqs. (28) and (29) Eq. (31) for the densities simplifies to

$$
\partial_{t_{2}} \rho_{s}=0
$$

This equation implies that the fluid is incompressible on the $t_{2}$ scale. In this case there is no diffusion on the $t_{2}$ scale because the ionic densities have already come to their equilibrium on the faster $t_{1}$ scale. On the other hand, if we assume that the second derivative of $\rho_{s}$ with respect to $t_{1}$ vanishes then Eq. (31) becomes a diffusion equation with the diffusion constant $D_{s}=c_{\mathrm{sv}}^{2} \gamma_{s} / 2$ for ions of type $s$. The diffusion process relaxes the densities $\rho_{s}(s=+,-)$ again to the equilibrium distribution (5).

We still have to discuss Eq. (32) for the mass current on the $t_{2}$ scale. It is reasonable to assume that the derivatives of $\rho_{s}$ and $\vec{j}_{s}$ with respect to $t_{1}$ are small on the $t_{2}$ scale. So we may neglect the first two terms in Eq. (32). Furthermore, we have to sum over $s$ on both sides of Eq. (32) in order to obtain the equation of motion for the total current $\vec{j}$ on the $t_{2}$ scale:

$$
\partial_{t_{2}} \vec{j}=\frac{1}{6} \nabla_{1}^{2} \vec{j}
$$


Moreover, if we combine this equation with Eq. (29) for variations on the $t_{1}$ scale we obtain the following equation:

$$
\partial_{t} \vec{j}=\frac{1}{6} \nabla^{2} \vec{j}-c_{\mathrm{sv}}^{2} \sum_{s} \gamma_{s} \nabla \rho_{s}-c_{\mathrm{sv}}^{2} \sum_{s} \gamma_{s} z_{s} \rho_{s} \nabla \hat{\Phi} .
$$

So we recover Eq. (8) for the total current whereby the kinematic viscosity $\nu$ is $1 / 6$ and the equation for the pressure depends on the parameters $\gamma_{s}$,

$$
p=c_{\mathrm{sv}}^{2} \sum_{s} \gamma_{s} \rho_{s}
$$

Note that for $\gamma_{1}=\gamma_{2}$ this is just the equation of state for an ideal gas.

\section{THE SEDIMENTATION VELOCITY}

In this section we present the results for the sedimentation velocity of an array of charged spheres in an electrolyte solution. We show that our method gives correct results in that it recovers an analytical formula which is valid in the limit of an isolated, weakly charged sphere in an unbounded electrolyte. Furthermore, we discuss the dependence of the sedimentation velocity on the charge of the spheres. We then compare our results to experimental data.

Before we show the results for the sedimentation velocity we discuss to what extent the calculated electrostatic potentials and the ionic densities around a macroion are influenced by lattice artifacts. The latter may have several reasons: We use a simple way to introduce the charge of a macroion. We assign its charge $Z$ by distributing the densities on the lattice nodes inside the macroion such that the net charge, i.e. the sum over all these nodes, is equal to $Z$. This may have the effect that the effective charge distribution on the surface of the macroion is anisotropic because the inner nodes form only an approximative representation of a sphere which can, of course, be improved by increasing its radius $a$. Another drawback which is due to the lattice lies in the range of the electrostatic potential. The Debye length, measured in lattice units, must be at least larger than one. Otherwise, the simulation becomes unstable because one has strong discontinuities in the ionic densities between two lattice points close to the surface of the macroion.

The importance of these artifacts can be checked by determining the radial ionic densities $\rho_{s}(r)$ around a spherical macroion, i.e. the densities for the ions of type $s$ at a distance $r$ from the center of the sphere. In the absence of artifacts $\rho_{s}(r)$ should obey

$$
\rho_{s}(r)=\bar{\rho}_{s} \exp \left(-z_{s} \hat{\Phi}(r)\right)
$$

In Fig. 1 1 we show an example of the $\rho_{s}(r)$ calculated directly (symbols) and from the righthand side of Eq. (37) (solid lines) for a macroion with a radius $a=4.5$ and charge $Z=100$. The length of the simulation box is $L=80$ and the density of the neutral fluid is set to $\rho_{n}=20$. The Debye length is varied by changing the sum of the mean ionic densities from $\sum_{s} \bar{\rho}_{s}=0.0063\left(\lambda_{D}=5.6\right)$ to $\sum_{s} \bar{\rho}_{s}=0.1\left(\lambda_{D}=1.4\right)$ whereby the Bjerrum length is $l_{B}=0.4$. We can infer from Fig. 1 that the ionic densities as calculated directly agree very well with those calculated from the right-hand side of Eq. (37) even for a Debye length 
as small as $\lambda_{D}=1.4$. This means that $a=4.5$ is a reasonable choice for the radius of a macroion which keeps lattice artifacts small at least for $\lambda_{D} \geq 1.4$.

Now we consider one charged macrosphere in a unit cell of length $L$ with a fixed position. It represents one particle in an array of spheres on a simple cubic lattice because it interacts with its own periodic images. The volume fraction of the macroions is given by $\varphi=\left(4 \pi a^{3}\right) /\left(3 L^{3}\right)$. In order to investigate sedimentation phenomena we have to introduce a gravitational force in the LB equations. After a time of the order $\tau_{\mathrm{s}}=L^{2} / \nu_{\text {eff }}\left(\nu_{\text {eff }}\right.$ : effective, kinematic viscosity of the fluid in the presence of the spheres) the steady state is reached for which one determines the average flow velocity in the unit cell. We divide the latter by the average flow velocity of the corresponding neutral system and yield the ratio $U / U_{0}$ of the sedimentation velocities in the charged and the neutral system, respectively. For the larger systems considered, we did not wait until the system came to its steady state, because we know that the time dependence of the apparent sedimentation velocity $U_{\text {app }}(t)$ is given by

$$
U_{\mathrm{app}}(t)=U\left(1-\exp \left(-\frac{t}{\tau_{\mathrm{s}}}\right)\right)
$$

By computing numerically the time derivative of Eq. (38) one obtains a simple exponential function with the two unknown quantities $U$ and $\tau_{s}$ which we computed from fits of the logarithm of this exponential function. With this procedure it was possible to determine $U$ within a time of the order $t \approx \tau_{\mathrm{s}} / 20$. We checked the accuracy of our fits at $\varphi=0.0018$ by comparing them to the exact steady state results, and we obtained identical results for $U / U_{0}$ as a function of $\kappa a\left(\kappa \equiv \lambda_{D}^{-1}\right)$.

In the following we show that our LB method recovers an analytical result for $U / U_{0}$ which was first derived by Booth [26], and later slightly modified by Ohshima et al. 27. [7 It is valid in the limit of infinite dilution and small charge of the macroions, i.e. a weakly charged macroion in an electrolyte with infinite extension. What do we expect in this case? Due to the external force the ionic concentrations around the macroions which form the electrical double layer deviate from their equilibrium values. The double layer loses its spherical symmetry due to the fluid motion which results in an electrical dipole field pointing in the direction opposite to the motion of the macroion and thus reduces its sedimentation velocity. Booth's calculation starts with the Ansatz

$$
\frac{U}{U_{0}}=1+\sum_{k=1}^{\infty} c_{k} Z^{k}
$$

and takes into account only terms in the lowest non-vanishing order in $Z^{k}$,

$$
\frac{U}{U_{0}}=1+c_{2} Z^{2}
$$

The coefficient $c_{2}$ can be calculated analytically by solving the electrokinetic equations of motion (1), (3), and (8) whereby the Poisson equation is solved in the Debye-Hückel limit. The final expression for $c_{2}$ has the following form [26,27]:

\footnotetext{
${ }^{1}$ However, Booth's result agrees with the one of Ohshima et al. for an 1-1-electrolyte. We are only interested in this special case in this paper.
} 


$$
c_{2}=-\frac{k_{B} T l_{B}}{72 \pi a^{2} \eta} \frac{\sum_{s} z_{s}^{4} \bar{\rho}_{s} D_{s}^{-1}}{\sum_{s} z_{s}^{2} \bar{\rho}_{s}} f(\kappa a) .
$$

$\bar{\rho}_{s}$ denotes the mean density of the ions of type $s$ far away from the center of the macroion. $\eta$ is the shear viscosity. $f(\kappa a)$ is a function of exponential integrals of different order $n$, $\mathrm{E}_{n}(x)=x^{n-1} \int_{x}^{\infty} d t t^{-n} \exp (-t)$ :

$$
\begin{aligned}
f(\kappa a)= & \frac{1}{1+(\kappa a)^{2}}\left[\mathrm{e}^{2 \kappa a}\left(3 E_{4}(\kappa a)-5 E_{6}(\kappa a)\right)^{2}+8 \mathrm{e}^{\kappa a}\left(E_{3}(\kappa a)-E_{5}(\kappa a)\right)\right. \\
& \left.-\mathrm{e}^{2 \kappa a}\left(4 E_{3}(2 \kappa a)+3 E_{4}(2 \kappa a)-7 E_{8}(2 \kappa a)\right)\right] .
\end{aligned}
$$

We have determined $U / U_{0}$ as a function of $\kappa a$ for different volume fractions. The radius of the macroion is fixed to $a=4.5$. Moreover, the diffusivities of the ionic species in the fluid are chosen to be $D_{1}=0.165$ for the counterions and $D_{2}=0.25$ for the coions. The ratio $D_{1} / D_{2}=0.66$ corresponds to that of $D_{\mathrm{Na}} / D_{\mathrm{Cl}}$ in sodium chloride. The Bjerrum length is again set to $l_{B}=0.4$. In order to vary $\kappa a$ from 0.15 to 1.5 we have to change $\sum_{s} \bar{\rho}_{s}$ from 0.00025 to 0.02211 , respectively. This is small compared to the density of the neutral fluid, $\rho_{n}=20$. So by changing $\kappa a$ we do not change the viscosity of the fluid significantly.

Fig. 2 shows the results for a surface charge $Z=10$ of the macroion. We demonstrate below that this value is small enough for the approximation (28) to hold. Firstly, we infer from Fig. 2 that the relative reduction of the sedimentation velocity due to the charges is only of the order of $10^{-4}$ at $Z=10$ in the $\varphi$-range considered. $U / U_{0}$ exhibits a minimum which moves to higher values of $\kappa a$ with increasing $\varphi$. The occurence of such a minimum is reasonable since an increase of $\kappa a$ is accompanied with two competing effects: On the one hand the electrostatic potential $\hat{\Phi}$ becomes stronger due to an increasing salt concentration but on the other hand it becomes also more short-ranged because of a decreasing Debye length, and thus, it affects only the flow nearby the macroion. The value of the minimum in $U / U_{0}$ decreases with decreasing $\varphi$ and seems to move towards the one of the Booth curve for $\varphi \rightarrow 0$. This also holds for the amplitude and the shape of $U / U_{0}$ for $\varphi \rightarrow 0$. In order to give quantitative evidence that our calculation would recover Booth's result we plot in the inset of Fig. $2 U / U_{0}$ as a function of $\varphi^{1 / 3}$ at $\kappa a=0.5$, i.e. around the position of the minimum. The fit in this figure with a straight line indeed approaches the Booth result, i.e. at $\varphi=0$.

Up to now we have shown only the results for $U / U_{0}$ for a small charge of the macroion $Z=10$. But it is of course interesting to check up to which values of $Z$ the approximation (40) holds. If Eq. (40) would be exact, one could renormalize $U / U_{0}$ as a function of $\kappa a$ for a given charge $Z=Z_{\text {old }}$ to a new charge $Z=Z_{\text {new }}$ by multiplying $1-U / U_{0}$ by $Z_{\text {new }}^{2} / Z_{\text {old }}^{2}$. In this way we have renormalized our data for $Z=10$ at $\varphi=0.00076$ to $Z=100$ and $Z=130$, and we compare these data sets in Fig. 3 a with the corresponding simulation results for the latter two values of $Z$. We see that we have strong corrections to the results as expected from Booth's theory, especially around the minimum in $U / U_{0}$. Firstly the amplitude of the minimum is underestimated by the renormalized curves and also the position of the minimum is at a slightly larger value. To study the corrections to Booth's theory more quantitatively we plot in Fig. $3 \mathrm{~b} 1-U / U_{0}$ as a function of $Z$ for $\kappa a=0.16,0.5$, and 1.5. The solid lines in this figure are fits of the form $g(Z)=c_{2} Z^{2}+c_{4} Z^{4}+c_{6} Z^{6}$. From the comparison of the different functions $g(Z)$ to the corresponding ones with only the leading term $\propto Z^{2}$ (dashed curves in Fig. 3 $3 \mathrm{~b}$ ) we can conclude that the corrections to Booth's theory become important for $Z>50$. 
Finally we address the question whether our results are in agreement with a dynamic light scattering experiment by Schumacher and van de Ven 28]. They measured the diffusion constant $D$ for a system of gold particles in distilled water. Note that $D / D_{0}$ is equal to $U / U_{0}$. The radius of the gold particles was around $20 \mathrm{~nm}$ and their volume fraction $2 \cdot 10^{-5} \%$. Different data sets were determined by changing the value of $\kappa a$ with different salts. We consider here the experimental data points measured with sodium chloride which are shown in Fig. $⿴$ in comparison to our simulation data at $\varphi=0.0046$ and at $\varphi=0.00076$ for $Z=100$. It is interesting that the experimental data can be very well described by the simulation curve for $\varphi=0.0046$ although the experiment was done at a very small volume fraction of gold particles and the curve for $\varphi=0.00076$ deviates strongly from the experimental data. More systematic experiments, e.g. for different volume fractions, would be necessary to clarify this discrepancy.

\section{CONCLUSIONS}

We have developed a LBM for the simulation of the dynamics of suspensions of charged colloidal particles. In this method a set of non-linear, coupled electrokinetic equations is solved which consists of convective diffusion equations for the ion densities $\rho_{s}(s=+,-)$, the linearized Navier-Stokes equations for the mass current $\vec{j}$, and the Poisson equation for the electrostatic potential $\hat{\Phi}$. Furthermore, a neutral fluid characterized by the density $\rho_{n}$ can be introduced in order to keep the viscosity in the fluid essentially constant. The propagation of $\rho_{s}$ and $\vec{j}$ is computed by means of one-particle distribution functions $n_{i}^{s}$ for each species $s$. But in contrast to the normal LBM $\rho_{s}$ and $\vec{j}$ are not simply given as zeroth and first moments of the $n_{i}^{s}$ 's, respectively. This is due to the coupling of the ionic part of the fluid to the gradient of $\hat{\Phi}$ which leads to an additional diffusive term in the propagation of the ion densities and a body force term in the propagation of the mass current $\vec{j}$. The electrostatic potential is determined from the ion densities by means of a Poisson equation solver for which we use a successive overrelaxation scheme. Our method is fast, stable and easy to implement. We have verified our numerical scheme by means of a Chapman-Enskog expansion.

As an application we applied our method to determine the reduced sedimentation velocity $U / U_{0}$ for an array of charged spheres on a simple cubic lattice. We determined $U / U_{0}$ as a function of the dimensionless parameter $\kappa a$ for different volume fractions of macroions $\varphi$. We compared our results with an analytical formula first derived by Booth which is valid in the limit of a weakly charged, isolated macroion in an unbounded electrolyte. Booth's theory starts with an expansion of $U / U_{0}$ in powers of the macroion charge $Z$ of which the lowest non-vanishing order, $Z^{2}$ is taken into account. We gave evidence that we recover Booth's result in the limit $\varphi \rightarrow 0$. For about $Z>50$ corrections to the Booth theory become important. Up to $Z=130$ our simulation data can be well described by an expansion up to order $Z^{6}$. These numerical data could be used to test theories that go beyond the Booth level. We mention that very recently also a mode coupling theory with hydrodynamic interactions was shown to be in agreement with Booth's theory [29]. This theory is also able to consider colloidal systems at finite volume fractions.

Our LBM for charged colloids is well suited to study their short-time dynamics and the flow around macroions. It is not restricted to steady state problems but one can also 
determine time dependent quantities like the velocity autocorrelation function of a tagged macroion in a colloidal suspension. Also particle shapes which are different from a spherical one can be easily introduced in our LBM. Moreover, the introduction of walls is rather simple which makes it possible to study charged colloidal particles in confined geometries.

Acknowledgments: We thank F. Capuani for a critical reading of the manuscript. The work of the FOM Institute is part of the scientific program of FOM and is supported by the Nederlandse Organisatie voor Wetenschappelijk Onderzoek (NWO). J. H. acknowledges financial support by the Deutsche Forschungsgemeinschaft (Grant HO 2231/1-1). 


\section{REFERENCES}

[1] M. J. Stevens, M. L. Falk, and M. O. Robbins, J. Chem. Phys. 104, 5209 (1996).

[2] E. Allahyarov, H. Löwen, and S. Trigger, Phys. Rev. E 57, 5818 (1998).

[3] R. Benzi, S. Succi, and M. Vergassola, Phys. Rep. 222, 145 (1992).

[4] A. J. C. Ladd, J. Fluid Mech. 271, 285 (1994); A. J. C. Ladd, J. Fluid Mech. 271, 311 (1994).

[5] S. Chen and G. D. Doolen, Ann. Rev. Fluid Mech. 30, 329 (1998).

[6] A. J. C. Ladd, Phys. Rev. Lett. 70, 1339 (1993).

[7] M. H. J. Hagen, D. Frenkel, and C. P. Lowe, J. Chem. Phys. 109, 275 (1998).

[8] M. H. J. Hagen, D. Frenkel, and C. P. Lowe, Physica A 272, 376 (1999).

[9] C. P. Lowe, D. Frenkel, and A. J. Masters, J. Chem. Phys. 103, 1582 (1995).

[10] M. W. Heemels, M. H. J. Hagen, and C. P. Lowe, J. Comp. Phys. 164, 48 (2000).

[11] M. H. J. Hagen, I. Pagonabarraga, C. P. Lowe, and D. Frenkel, Phys. Rev. Lett. 78, 3785 (1997).

[12] I. Pagonabarraga, M. H. J. Hagen, C. P. Lowe, and D. Frenkel, Phys. Rev. E 58, 7288 (1998).

[13] I. Pagonabarraga, M. H. J. Hagen, C. P. Lowe, and D. Frenkel, Phys. Rev. E 59, 4458 (1999).

[14] U. Oxaal, E. G. Flekkoy, and J. Feder, Phys. Rev. Lett. 72, 3514 (1994).

[15] A. Koponen, D. Kandhai, E. Hellen, M. Alava, A. Hoekstra, M. Kataja, K. Niskanen, P. Sloot, and J. Timonen, Phys. Rev. Lett. 80, 716 (1998).

[16] C. P. Lowe and D. Frenkel, Phys. Rev. Lett. 77, 4552 (1996).

[17] D. L. Koch, R. J. Hill, and A. S. Sangani, Phys. Fluids 10, 3035 (1998).

[18] P. Ahlrichs and B. Dünweg, J. Chem. Phys. 111, 8225 (1999).

[19] X. He and N. Li, Comp. Phys. Comm. 129, 158 (2000).

[20] P. B. Warren, Int. J. Mod. Phys. C 8, 889 (1997).

[21] D. Frenkel and M. H. Ernst, Phys. Rev. Lett. 63, 2165 (1989).

[22] R. J. Hunter, Foundations of Colloid Science, Vol. II (Clarendon Press, Oxford, 1989).

[23] W. H. Press, S. A. Teukolsky, W. T. Vetterling, and B. P. Flannery, Numerical Recipes (Cambridge University Press, Cambridge, 1992).

[24] M. M. Gupta, J. Kouatchou, and J. Zhang, J. Comp. Phys. 132, 226, (1997); J. Zhang, J. Comp. Phys. 143, 449 (1998).

[25] U. Frisch, B. Hasslacher, and Y. Pomeau, Phys. Rev. Lett. 56, 1505 (1986).

[26] F. Booth, J. Chem. Phys. 22, 1956 (1954).

[27] H. Ohshima, T. W. Healy, L. R. White, and R. W. O'Brien, J. Chem. Soc., Faraday Trans. 80, 1299 (1984).

[28] G. A. Schumacher and T. G. M. van de Ven, Faraday Discuss. Chem. Soc. 83, 75 (1987).

[29] M. Kollmann and G. Nägele, Europhys. Lett. 52,474 (2000); M. Kollmann and G. Nägele, J. Chem. Phys. 113, 7672 (2000). 


\section{FIGURES}

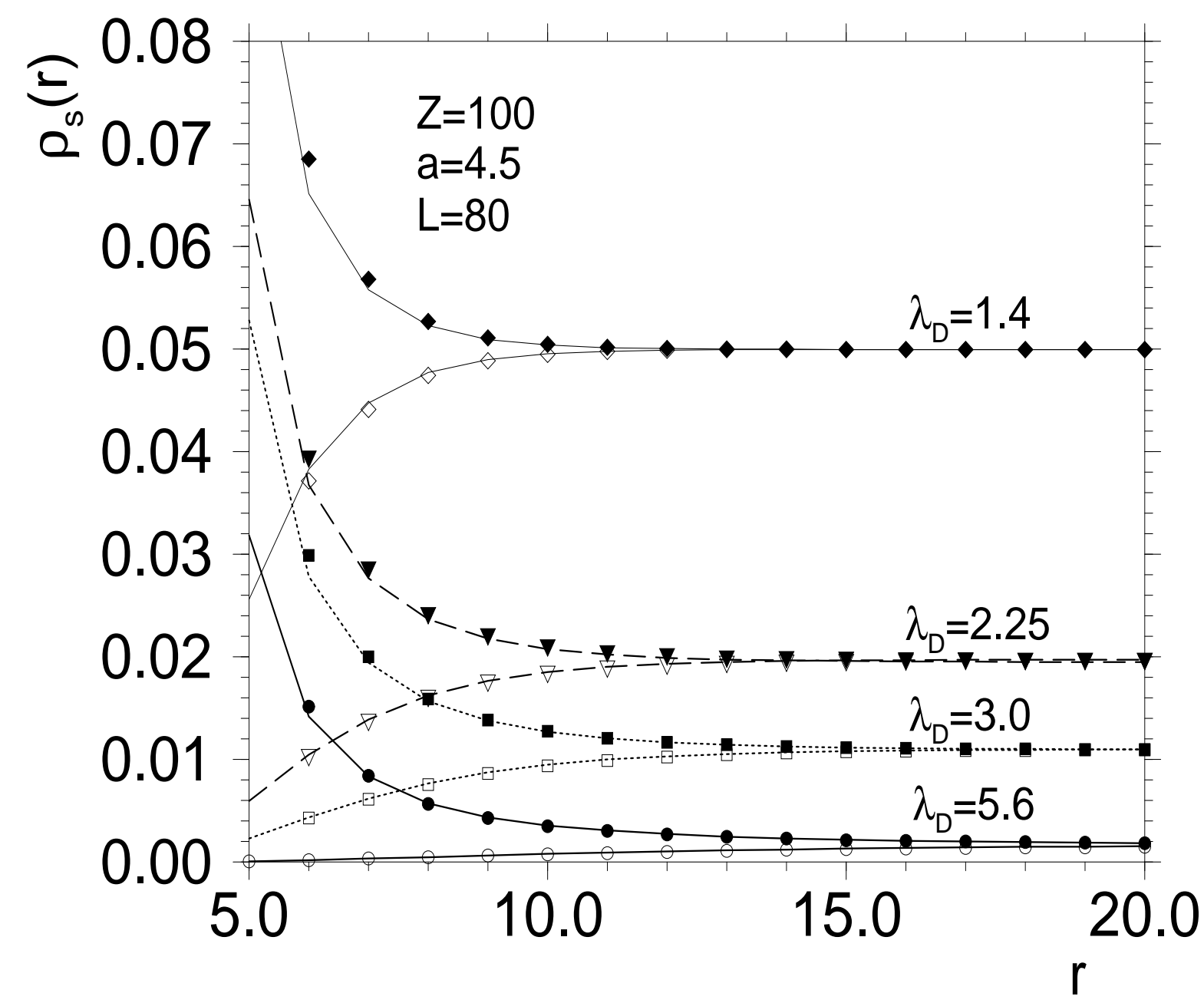

FIG. 1. The radial ionic densities around a macroion with $a=4.5$ for $Z=100$ as determined directly from $\rho_{s}(\vec{r})$ (filled symbols for the counterions and open symbols for the coions) and by calculating it from the electrostatic potential (lines) — see text — for the indicated Debye lengths $\lambda_{D}$. 


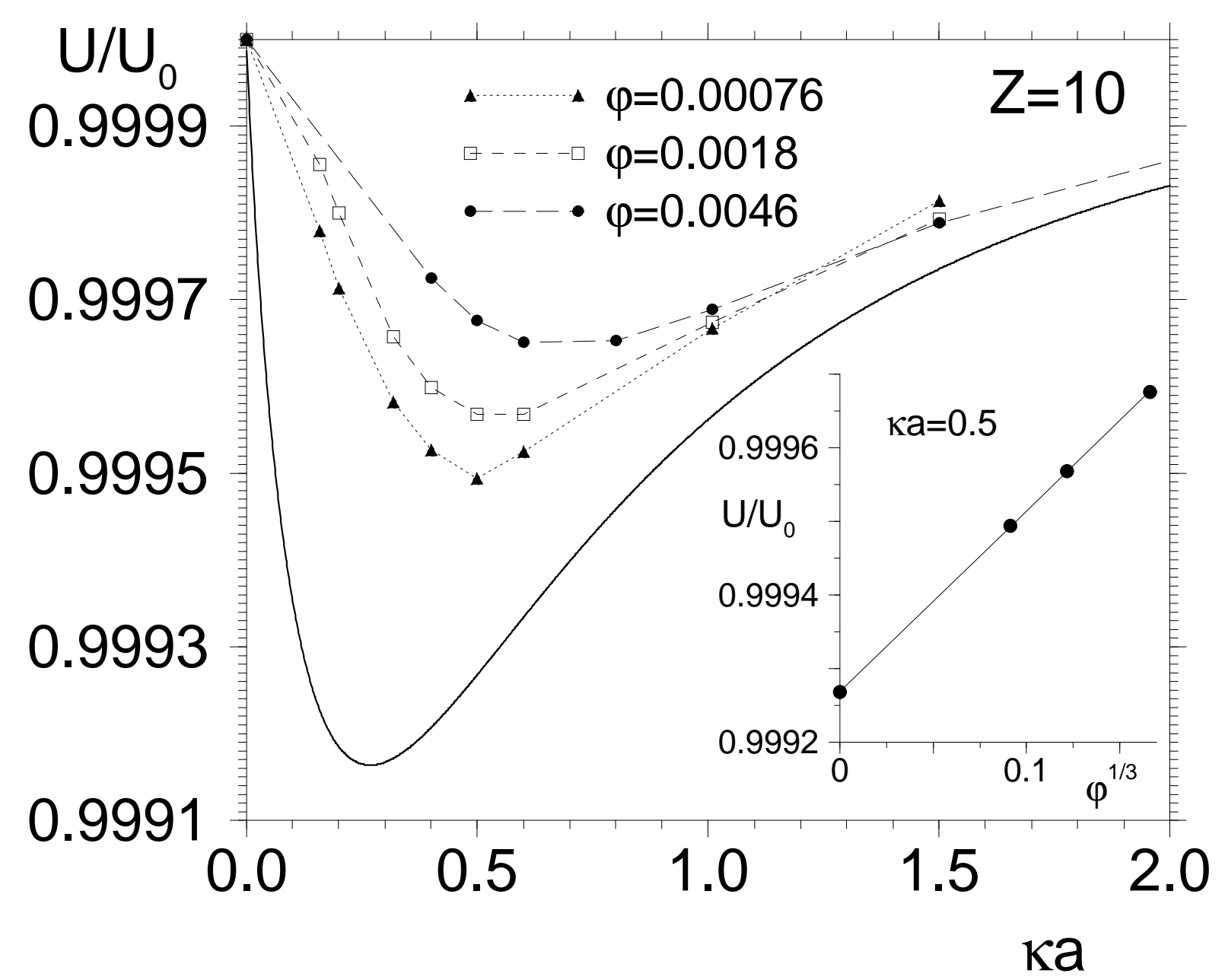

FIG. 2. $U / U_{0}$ for the volume fractions $\varphi=0.0046,0.0018$, and 0.00076 as a function of $\kappa a$. The charge of the macroion is set to $Z=10$. The solid line is the result from Booth's theory. The inset shows $U / U_{0}$ as a function of $\varphi^{1 / 3}$ for $\kappa a=0.5$. The solid line in the inset is the fit function $0.999269+0.0024556 \varphi^{1 / 3}$. 

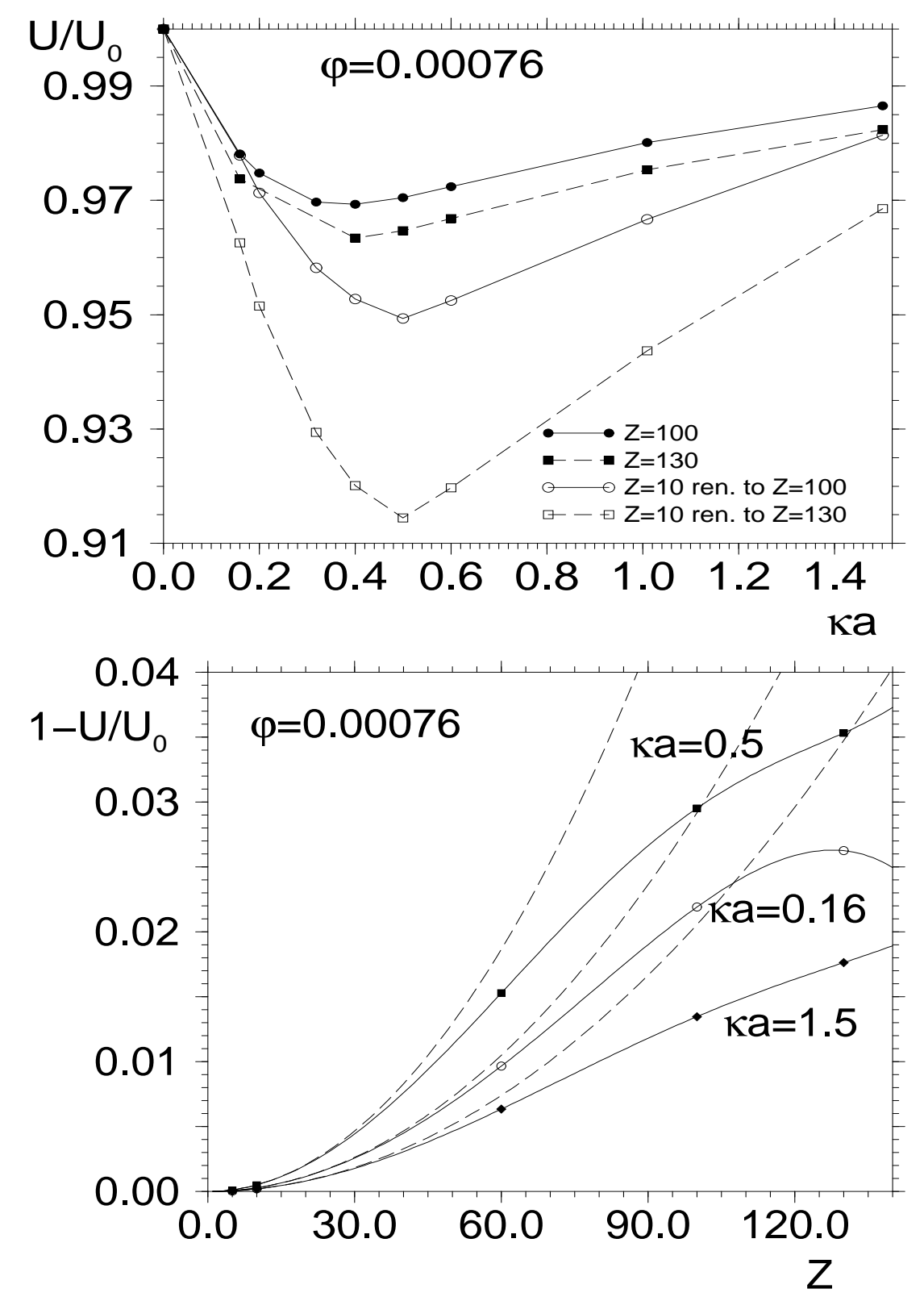

FIG. 3. a) $U / U_{0}$ at $\varphi=0.00076$ as a function of $\kappa a$ for $Z=100$ and $Z=130$ (filled symbols). The open symbols show data for $Z=10$ which are renormalized to $Z=100$ and $Z=130$ (see text). b) $1-U / U_{0}$ as a function of $Z$ for the indicated values of $\kappa a$. The solid lines show the following fit functions: $g(Z)=2.916 \cdot 10^{-6} Z^{2}-6.086 \cdot 10^{-11} Z^{4}-1.173 \cdot 10^{-15} Z^{6}$ for $\kappa a=0.16, g(Z)=5.182 \cdot 10^{-6} Z^{2}-2.810 \cdot 10^{-10} Z^{4}+5.801 \cdot 10^{-15} Z^{6}$ for $\kappa a=0.5$, $g(Z)=2.056 \cdot 10^{-6} Z^{2}-8.684 \cdot 10^{-11} Z^{4}+1.592 \cdot 10^{-15} Z^{6}$ for $\kappa a=1.5$. The dashed lines show only the term $\propto Z^{2}$ of the latter three functions. 


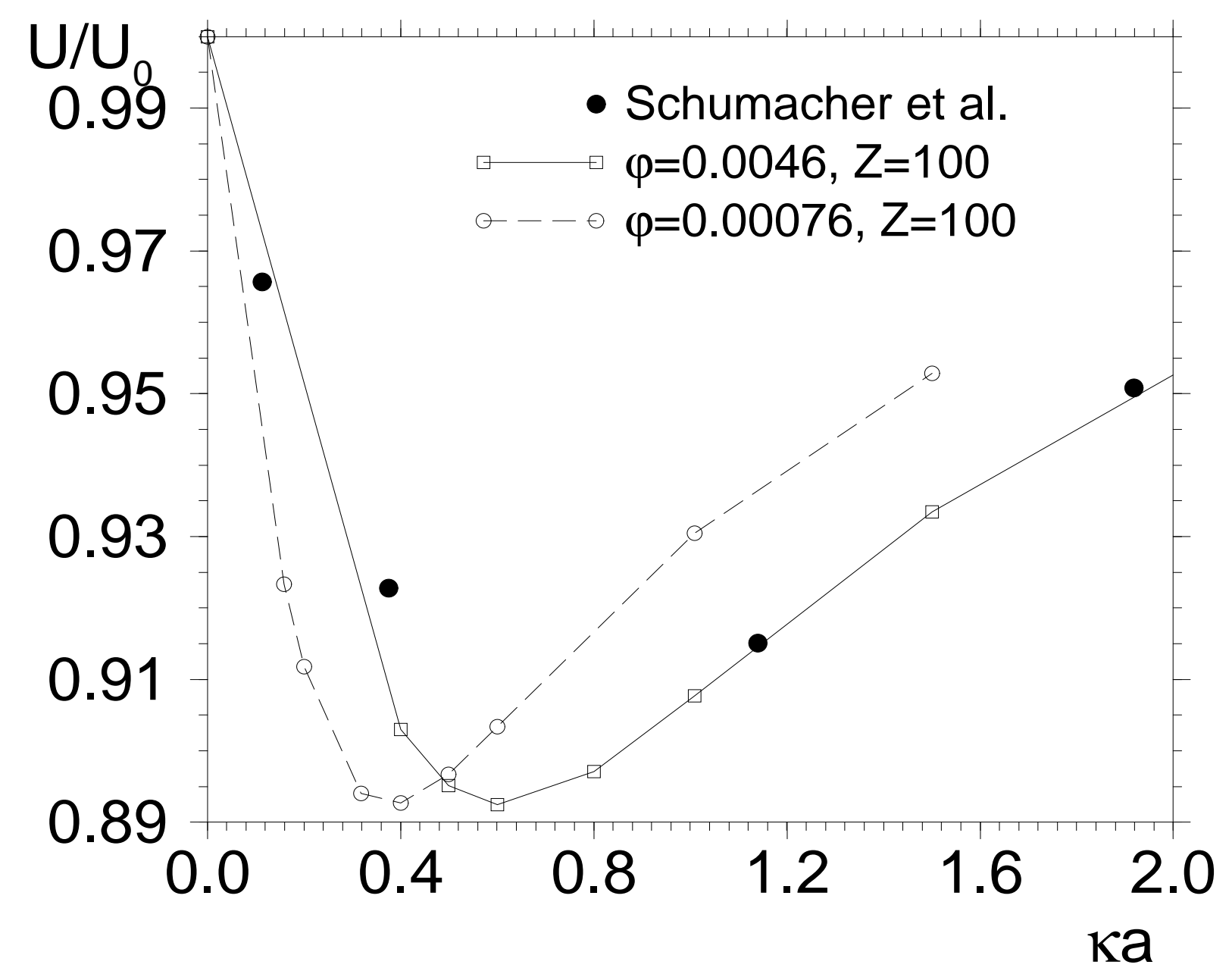

FIG. 4. $U / U_{0}$ at $\varphi=0.00076$ and $\varphi=0.0046$ as a function of $\kappa a$ for $Z=100$ in comparison to experimental data (closed circles). 